\section{SISTEMAS DE PRODUCCIÓN DE FORRAJE PARA INCREMENTAR LA PRODUCTIVIDAD DEL AGUA}

\section{FORAGE CROPPING SYSTEMS FOR INCREASING WATER PRODUCTIVITY}

\author{
David G. Reta Sánchez ${ }^{1 *}$, Uriel Figueroa \\ Viramontes $^{1}$, Rodolfo Faz Contreras ${ }^{1}$, Gregorio \\ Núñez Hernández ${ }^{1}$, Arturo Gaytán Mascorro ${ }^{1}, \mathbf{J}$. \\ Santos Serrato Corona ${ }^{2}$ y Jesús A. Payán García ${ }^{3}$
}

\begin{abstract}
${ }^{1}$ Campo Experimental La Laguna, Instituto Nacional de Investigaciones Forestales, Agrícolas y Pecuarias (INIFAP). Blvd. José Santos Valdez 1200, Col. Centro. 27440, Cd. Matamoros, Coahuila. ${ }^{2}$ Facultad de Agricultura y Zootecnia, Universidad Juárez del Estado de Durango. Domicilio Conocido, Ejido. Venecia, Dgo. Apdo. Postal 1-142. 35111, Gómez Palacio, Dgo. ${ }^{3}$ Campo Experimental Delicias, INIFAP. Km. 2 Carretera Delicias-Rosales, Col. Centro. 33000, Cd. Delicias, Chih.
\end{abstract}

* Autor para correspondencia (reta.david@inifap.gob.mx)

\section{RESUMEN}

La incorporación de especies alternativas en los sistemas de producción forrajeros puede mejorar la productividad del agua (PA). El objetivo del estudio fue comparar el rendimiento de forraje y la PA de tres sistemas de especies alternativas respecto a dos sistemas tradicionales en la Comarca Lagunera, México. En cada especie se determinó la calidad de forraje y se estimaron los rendimientos y la PA en la producción de materia seca (MS), proteína cruda (PC) y energía neta para lactancia (ENL). Los rendimientos de MS y la ENL de la chicoria (Cichorium intybus L.) fueron similares a los de alfalfa (Medicago sativa $\mathbf{L}$.), pero con menor concentración y rendimiento de PC. El mejor sistema alternativo con especies de ciclo anual, incluyó canola (Brassica napus L.), maíz (Zea mays L.) y soya (Glycine max L. Merr.). Este sistema, con un volumen de agua aplicado similar al de alfalfa, produjo rendimientos de $\mathrm{PC}$ iguales $(\mathrm{P}>\mathbf{0 . 0 5})$ a los de alfalfa, pero con mayores rendimientos de MS (62 \%) y ENL (77 \%); por tanto, los valores de la PA en producción de MS y ENL se incrementaron en 60 y $75 \%$, respectivamente.

Palabras clave: Calidad de forraje, especies alternativas, rendimiento de materia seca.

\section{SUMMARY}

Incorporation of alternative species into forage cropping systems may increase water productivity (WP). The objective of this study was to compare forage production and WP of three alternative cropping systems in comparison with two conventional systems in the Comarca Lagunera, México. Forage quality in each species was determined, and yields and WP in dry matter (DM), crude protein (CP) and net energy for lactation (NEL) production were estimated. Chicory (Cichorium intybus L.) produced similar DM and NEL yields than alfalfa (Medicago sativa $\mathrm{L}$.), but with lower concentration and yield of $\mathrm{CP}$. The alternative system, which included canola (Brassica napus L.), corn (Zea mays L.) and soybean (Glycine max L. Merr.), was the best choice among those with annual forages. Compared to alfalfa, this cropping system with a similar volume of water applied, produced equivalent $(P>0.05)$ CP yields, but higher yields of DM (62 \%) and NEL (77 \%); thus WP values in DM and NEL production increased by 60 and $75 \%$, respectively.

Index words: Forage quality, alternative species, dry matter yield.

\section{INTRODUCCIÓN}

La producción de leche de ganado bovino es una de las principales actividades económicas en la Comarca Lagunera, México, por lo que existe una alta demanda de forraje de calidad, el cual se produce en condiciones de riego pero con escasa disponibilidad de agua. La producción de forraje se basa en pocas especies, como maíz (Zea mays L.), sorgo (Sorghum bicolor L. Moench), cereales de invierno y especialmente alfalfa (Medicago sativa L.), que ocupa $40 \%$ de superficie total forrajera. La alfalfa es una leguminosa cuyo forraje es de alta calidad (Putnam et al., 2008) y tiene un papel importante en la rotación de cultivos (Russell et al., 2006); sin embargo, la productividad del agua (PA) de esta especie es baja (1.18 $\mathrm{kg}$ de MS por $\mathrm{m}^{3}$ de agua aplicada) (Quiroga y Faz, 2008).

Una opción poco explorada para incrementar la PA es incorporar especies alternativas a los sistemas de producción forrajeros. En un estudio previo (Reta et al., 2008), los cultivos alternativos que sobresalieron por su rendimiento, calidad de forraje y PA en la Comarca Lagunera fueron canola (Brassica napus L.) y chícharo (Pisum sativum L.) en el ciclo otoño-invierno, y soya (Glycine $\max$ L. Merr.) en primavera-verano. Como especie de cortes múltiples, la chicoria (Cichorium intybus L.) tuvo buen rendimiento de materia seca y calidad de forraje. El objetivo del presente estudio fue comparar el rendimiento de forraje y la productividad del agua de tres sistemas de especies alternativas respecto a dos sistemas tradicionales en la Comarca Lagunera, México.

\section{MATERIALES Y MÉTODOS}

El estudio se realizó de noviembre de 2007 a noviembre de 2008 en Matamoros, Coahuila, México $\left(25^{\circ} 32^{\prime} \mathrm{LN}\right.$, $103^{\circ} 14^{\prime}$ LO y $1150 \mathrm{msnm}$ ), en un suelo de textura franco arcilloso. Se estudiaron tres sistemas de especies alternativas, con especies para los ciclos de invierno, primavera y verano: (1) canola-maíz-soya; (2) chícharomaíz-soya; y (3) chicoria (unicultivo) como cultivo perenne. Los testigos fueron: (4) alfalfa (unicultivo) y (5) 
avena-maíz-maíz. Se utilizó un diseño experimental de bloques completos al azar con cuatro repeticiones. Las parcelas experimentales fueron de $6 \mathrm{~m}$ de ancho por $20 \mathrm{~m}$ de longitud; la parcela útil fue de $24 \mathrm{~m}^{2}$, ubicada en la parte central de cada unidad experimental.

En el ciclo otoño-invierno, la alfalfa, chicoria, avena, canola y chícharo se sembraron en seco el 9 de noviembre de 2007; el riego de siembra se aplicó al día siguiente. La distancia entre surcos fue $0.20 \mathrm{~m}$ en todos los cultivos, excepto en chícharo que se estableció en surcos a $0.38 \mathrm{~m}$. Se fertilizó con sulfato de amonio y fosfato monoamónico granulados. Para facilitar la emergencia de plantas, $9 \mathrm{~d}$ después de la siembra (dds) se aplicó un riego ligero (8 cm de lámina) en estas especies.

En alfalfa se sembró la variedad 'Excelente'; se fertilizó sólo en la siembra con $43 \mathrm{~kg}$ de $\mathrm{N}$ y $200 \mathrm{~kg}$ de $\mathrm{P}_{2} \mathrm{O}_{5} \mathrm{ha}^{-1}$; se le aplicaron cinco riegos cada $17 \mathrm{~d}$ para el primer corte y 11 riegos entre el primero y último corte para un total de ocho cortes entre el 4 de marzo del 2007 y el 5 de noviembre del 2008. Todos hechos al inicio de floración. En chicoria se sembró la variedad 'Puna' con una dosis total de fertilización durante el año de $876 \mathrm{~kg} \mathrm{~N} \mathrm{ha}^{-1}$ y de $200 \mathrm{~kg}$ de $\mathrm{P}_{2} \mathrm{O}_{5} \mathrm{ha}^{-1}$; en la siembra se aplicaron $43 \mathrm{~kg} \mathrm{~N}$ $\mathrm{ha}^{-1} \mathrm{y}$ todo el fósforo; posteriormente se fertilizó con 114 $\mathrm{kg} \mathrm{N} \mathrm{ha}^{-1}$ antes del primer corte y $120 \mathrm{~kg} \mathrm{~N} \mathrm{ha}^{-1}$ antes de la cosecha en los seis cortes posteriores; el calendario de riegos fue igual al de alfalfa, para un total de siete cortes del $1^{\circ}$ de abril al 5 de noviembre de 2008, hechos cuando las plantas tenían $40 \mathrm{~cm}$ de altura.

De avena se sembró la variedad 'Cuauhtémoc' con una dosis de fertilización de $260 \mathrm{~kg} \mathrm{~N}^{-1}$ y de $80 \mathrm{~kg}$ de $\mathrm{P}_{2} \mathrm{O}_{5}$ $\mathrm{ha}^{-1}$; en la siembra se aplicaron $70 \mathrm{~kg} \mathrm{~N} \mathrm{ha}^{-1}$ y todo el fósforo, y posteriormente se fertilizó con 130 y $60 \mathrm{~kg} \mathrm{~N}$ $\mathrm{ha}^{-1}$ en el primero y segundo riego, respectivamente; se aplicaron tres riegos, a los 48, 77 y 103 dds; la cosecha fue a los 117 dds (formación del grano). En cuanto a canola se sembró la variedad 'IMC 205', con dosis de fertilización y épocas de aplicación iguales a la avena; se le aplicaron dos riegos a los 48 y $77 \mathrm{dds}$; la cosecha fue a los 100 dds (floración e inicio de crecimiento de vainas). En chícharo se sembró la variedad 'Secada Laguna', con dosis de fertilización y épocas de aplicación iguales a avena y canola, y un calendario de riegos igual al de avena; se cosechó a los 117 dds (llenado de vainas).

En el ciclo de primavera, en las parcelas donde se cosechó canola, chícharo y avena, se sembró el híbrido de maíz ' $3025 \mathrm{~W}$ ' en suelo húmedo, el 14 de marzo de 2008 a una distancia entre surcos de $0.76 \mathrm{~m}$ y una densidad de población de 96629 plantas ha ${ }^{-1}$. La dosis de fertilización fue $300 \mathrm{~kg} \mathrm{~N}$ y $100 \mathrm{~kg} \mathrm{P}_{2} \mathrm{O}_{5} \mathrm{ha}^{-1}$. En la siembra se aplicaron $50 \mathrm{~kg} \mathrm{~N} \mathrm{ha}^{-1}$ y todo el fósforo; posteriormente se fertilizó con $150 \mathrm{~kg}$ y $100 \mathrm{~kg} \mathrm{~N}$ ha ${ }^{-1}$ en el primero y segundo riego, respectivamente; las fuentes fueron sulfato de amonio y fosfato monoamónico granulados. Se aplicó un riego de presiembra y cuatro riegos a los 40, 61, 77 y 94 dds. El maíz se cosechó a los 112 dds, en la fase de grano de un tercio de la línea de leche.

En el ciclo de verano, en las parcelas donde se estableció avena y maíz en invierno y primavera, respectivamente, se sembró maíz; mientras que en las parcelas con canola y chícharo en invierno y maíz en primavera, se estableció soya. La siembra en las dos especies fue en suelo húmedo el 29 de julio de 2008. En maíz se sembró el híbrido 'DK 2000' en surcos a $0.76 \mathrm{~m}$ con una densidad de población de 106600 plantas ha $^{-1}$; la dosis de fertilización fue $230 \mathrm{~kg} \mathrm{~N}^{-1}$ y $100 \mathrm{~kg} \mathrm{\textrm {P } _ { 2 } \mathrm { O } _ { 5 }}$ $\mathrm{ha}^{-1}$; en la siembra se aplicaron $50 \mathrm{~kg} \mathrm{~N}^{-1}$ y todo el fósforo; en el primero y segundo riego se fertilizó con 120 y $60 \mathrm{~kg} \mathrm{~N} \mathrm{ha}^{-1}$, respectivamente; se aplicó un riego de presiembra y tres riegos a los 32,52 y $70 \mathrm{dds}$; la cosecha fue a los 93 dds en la fase de grano masoso. En soya se sembró la variedad 'Hutchinson' en surcos a $0.76 \mathrm{~m}$, con una densidad de población de 375162 plantas ha ${ }^{-1}$; la dosis de fertilización fue $250 \mathrm{~kg} \mathrm{~N}$ y $100 \mathrm{~kg} \mathrm{P}_{2} \mathrm{O}_{5} \mathrm{ha}^{-1}$; en la siembra se aplicaron $70 \mathrm{~kg} \mathrm{~N} \mathrm{ha}^{-1} \mathrm{y}$ todo el fósforo; el manejo de la fertilización y los riegos durante el ciclo fue igual que en maíz en dosis y épocas de aplicación, con sulfato de amonio y fosfato monoamónico granulados como fuentes. El control de plagas en todos los cultivos fue mediante la aplicación de insecticidas durante el ciclo, y el de maleza en forma manual y con azadón.

A la cosecha en todas las especies se midió el rendimiento de forraje fresco, y en una muestra por parcela se obtuvo el porcentaje de materia seca y la calidad del forraje. La muestra fue de $1 \mathrm{~m}^{2}$ en alfalfa, chicoria, avena, canola, chícharo y maíz, y de $2.3 \mathrm{~m}^{2}$ en soya. Estas plantas se secaron en una estufa de aire forzado a $60^{\circ} \mathrm{C}$ hasta peso constante. El rendimiento de MS se estimó mediante el producto del rendimiento de forraje fresco por el porcentaje de MS. Las plantas secas se molieron en un molino Wiley ${ }^{\circledR}$ (Thomas Scientific, Swedesboro, NJ, EUA) con malla de $1 \mathrm{~mm}$; con los procedimientos descritos por Goering y Van Soest (1970) se determinó fibra detergente neutro (FDN) y fibra detergente ácido (FDA), y con el método de Kjeldahl para N (Bremner, 1996). Los contenidos de energía neta para lactancia (ENL), carbohidratos no fibrosos (CNF) y total de nutrientes digestibles (TND) se estimaron de acuerdo con las metodologías del Consejo Nacional de Investigación (NRC, 2001). Los rendimientos de proteína cruda (PC) y ENL por hectárea se obtuvieron al multiplicar los contenidos de PC y ENL por el 
rendimiento de MS de cada especie. La PA en cada sistema de especies se estimó con el cociente del rendimiento de MS, PC y ENL entre el volumen total de agua recibido por cada sistema de producción, el cual se estimó de la siguiente forma: volumen de agua aplicada + (lluvia acumulada - evaporación en cada evento durante el periodo del estudio).

Para medir el agua aplicada en los sistemas de producción se construyó un aforador tipo Parshall de lámina galvanizada. La ecuación para determinar el caudal aplicado fue la siguiente: $\mathrm{Q}=\mathrm{KH}^{\mathrm{n}}$, donde: $\mathrm{Q}=$ caudal en $\mathrm{m}^{3} \mathrm{seg}^{-1} ; \mathrm{H}=$ carga medida aguas arriba de la garganta, en metros; $\mathrm{n}=$ exponente (1.58); $\mathrm{K}=$ factor que depende del ancho de la garganta $(15.24 \mathrm{~cm}=1.58)$ (Hudson, 1997).

Se hicieron análisis de varianza con las variables: rendimiento, volumen de agua aplicado y productividad del agua en la producción de materia seca y nutrientes ( $\mathrm{P}$ $\leq$ 0.05) de los cinco sistemas de especies. No se realizaron análisis de varianza para calidad de forraje, pues los datos se obtuvieron en tres distintos ciclos de desarrollo; sin embargo, se hizo un análisis de correlación $(\mathrm{P} \leq 0.05)$ entre todas las características de calidad de forraje. Para comparar las medias se utilizó la prueba de Tukey $(\mathrm{P} \leq 0.05)$. Los datos se analizaron con el programa estadístico SAS (SAS Institute, 1985).

\section{RESULTADOS Y DISCUSIÓN}

\section{Calidad de forraje}

Los análisis de correlación (no presentados) indicaron que la PC no correlacionó con las demás características de la calidad de forraje evaluadas ( $\mathrm{P} \leq 0.05)$, mientras que la ENL correlacionó negativamente con la FDA $(r=-0.68$;
$P \leq 0.05)$. La alfalfa fue la especie convencional que presentó el forraje de mejor calidad. El maíz presentó un bajo nivel de PC, pero su valor energético (5.9 $\mathrm{MJ} \mathrm{kg}^{-1} \mathrm{de}$ MS) fue bueno, similar al promedio obtenido por Núñez et al. (2006). La avena cosechada en la etapa de formación de grano tuvo un contenido de PC de $134 \mathrm{~g}$ $\mathrm{kg}^{-1}$, con mayor contenido fibroso y menor concentración de ENL que maíz (Cuadro 1).

La calidad de forraje de chicoria fue inferior a la de alfalfa, con menores contenidos de PC, TND y ENL. Esta calidad fue menor a la obtenida en ciclos anteriores en la región (Reta et al., 2008), debido probablemente a que en el presente estudio hubo mayor desarrollo de tallos florales a la cosecha. Canola y chícharo tuvieron altos contenidos de PC, menor concentración de fibras que avena, especialmente la canola, y un contenido energético similar al de avena. Sólo la canola produjo forraje con valores de parámetros de calidad similares a la alfalfa. La soya durante el verano tuvo mejores valores de PC, fibras y ENL que el maíz. En comparación con la alfalfa, la calidad de soya fue similar, con excepción de PC, cuyo valor de $137 \mathrm{~g} \mathrm{~kg}^{-1}$ (Cuadro 1) fue menor que en ciclos anteriores cuando se registraron valores de $240 \mathrm{~g} \mathrm{~kg}^{-1}$ (Reta et al., 2008), pues el cultivo presentó hojas cloróticas durante una parte del ciclo debido a deficiencias de Fe.

De acuerdo con Putnam et al. (2008), alfalfa y canola son forrajes con buen valor energético y proteico. En este estudio el maíz produjo forraje bajo en proteína y alto en energía; la avena fue regular en proteína y energía; la soya fue alta en energía y regular en proteína. La chicoria fue un forraje alto en proteína pero con bajo valor energético, y el chícharo se consideró bueno en proteína y de regular a bajo en energía.

Cuadro 1. Características de calidad de forraje de las especies establecidas en cinco sistemas de producción evaluados en la Comarca Lagunera, 2007-2008.

\begin{tabular}{|c|c|c|c|c|c|c|c|}
\hline Cultivos & Ciclo & $\mathrm{PC}$ & FDA & $\begin{array}{c}\text { FDN } \\
\left(\mathrm{g} \mathrm{kg}^{-1}\right)\end{array}$ & $\mathrm{CNF}$ & TND & $\begin{array}{c}\text { ENL } \\
\left(\mathrm{MJ} \mathrm{kg}^{-1} \mathrm{MS}\right)\end{array}$ \\
\hline Alfalfa & Perenne & 220 & 336 & 425 & 214 & 560 & 5.69 \\
\hline Chicoria & Perenne & 159 & 392 & 394 & 229 & 444 & 3.97 \\
\hline Avena & Invierno & 134 & 392 & 620 & 148 & 562 & 5.40 \\
\hline Canola & Invierno & 230 & 355 & 398 & 202 & 561 & 5.69 \\
\hline Chícharo & Invierno & 176 & 427 & 526 & 184 & 540 & 5.23 \\
\hline Maíz & Primavera & 85 & 291 & 488 & 361 & 648 & 5.90 \\
\hline Maíz & Verano & 76 & 346 & 561 & 291 & 615 & 5.65 \\
\hline Soya & Verano & 137 & 330 & 403 & 271 & 606 & 5.82 \\
\hline
\end{tabular}


Cuadro 2. Volumen de agua aplicada, rendimiento, y productividad del agua (PA) en la producción de materia seca (MS), proteína cruda (PC) y energía neta para lactancia (ENL) en cinco sistemas de producción evaluados en la Comarca Lagunera, 2007-2008.

\begin{tabular}{|c|c|c|c|c|c|c|c|}
\hline \multirow{3}{*}{$\begin{array}{l}\text { Patrones } \\
\text { Inv-Prim-Ver }\end{array}$} & \multirow{3}{*}{$\begin{array}{c}\text { Agua aplicada } \\
\left(\mathrm{m}^{3} \mathrm{ha}^{-1}\right)\end{array}$} & \multicolumn{3}{|c|}{ Rendimiento de } & \multicolumn{3}{|c|}{ PA } \\
\hline & & MS & PC & \multirow{2}{*}{$\begin{array}{c}\text { ENL } \\
\left(\mathrm{MJ} \mathrm{ha}^{-1}\right)\end{array}$} & MS & $\mathrm{PC}$ & \multirow{2}{*}{$\begin{array}{c}\mathrm{ENL} \\
\left(\mathrm{MJ} \mathrm{m}^{-3}\right)\end{array}$} \\
\hline & & \multicolumn{2}{|c|}{$\left(\mathrm{kg} \mathrm{ha}^{-1}\right)$} & & \multicolumn{2}{|c|}{$\left(\mathrm{kg} \mathrm{m}^{-3}\right)$} & \\
\hline Alfalfa & $19237 \mathrm{~b}^{\dagger}$ & $19200 \mathrm{~d}$ & $4234 \mathrm{a}$ & $102906 \mathrm{~d}$ & $1.00 \mathrm{~d}$ & $0.22 \mathrm{a}$ & $5.35 \mathrm{~d}$ \\
\hline Chicoria & $19196 \mathrm{~b}$ & $21349 \mathrm{~d}$ & $3490 \mathrm{c}$ & $96248 \mathrm{~d}$ & $1.11 \mathrm{~d}$ & $0.18 \mathrm{bc}$ & $5.01 \mathrm{~d}$ \\
\hline $\mathrm{Av}-\mathrm{Mz}-\mathrm{Mz}$ & $20420 \mathrm{a}$ & $40796 \mathrm{a}$ & $3689 \mathrm{bc}$ & $234104 \mathrm{a}$ & $2.00 \mathrm{a}$ & $0.18 \mathrm{bc}$ & $11.46 \mathrm{a}$ \\
\hline Cn-Mz-Sy & 19492 b & $31191 \mathrm{~b}$ & $4032 a b$ & $182364 \mathrm{~b}$ & $1.60 \mathrm{~b}$ & $0.21 \mathrm{ab}$ & $9.36 \mathrm{~b}$ \\
\hline Ch-Mz- Sy & $20381 \mathrm{a}$ & $27678 \mathrm{c}$ & $3262 \mathrm{c}$ & $157831 \mathrm{c}$ & $1.36 \mathrm{c}$ & $0.16 \mathrm{c}$ & $7.74 \mathrm{c}$ \\
\hline
\end{tabular}

${ }^{\dagger}$ Medias en cada columna seguidas con la misma letra no son diferentes (Tukey, 0.05). ${ }^{\ddagger} \mathrm{Inv}=$ invierno; Prim $=$ primavera; Ver $=\mathrm{verano;}$ Av $=$ avena; $\mathrm{Mz}=$ maíz; $\mathrm{Cn}=$ canola; $\mathrm{Sy}=$ soya Ch = chícharo.

\section{Rendimiento y productividad del agua (PA)}

Los volúmenes de agua aplicados en alfalfa y chicoria fueron similares, mientras que en los sistemas con tres especies los volúmenes variaron de 19492 a $20420 \mathrm{~m}^{3}$ ha $^{-1}$; el menor correspondió al sistema canola-maíz-soya. Los rendimientos y la productividad del agua para la producción de MS y ENL de chicoria fueron similares a los de alfalfa; sin embargo, los valores del rendimiento y la PA en la producción de PC fueron inferiores en chicoria (Cuadro 2), debido a su menor concentración de proteína (Cuadro 1).

En comparación con las especies perennes, los sistemas de producción con tres especies por año presentaron los mayores rendimientos de forraje y de productividad del agua en cuanto a MS y ENL, lo cual se debe a que las especies anuales se desarrollan bajo condiciones de clima más cercanas a las óptimas. En esta región el maíz presenta mayor eficiencia de producción de MS que la alfalfa y chicoria por su mejor adaptación a las altas temperaturas durante primavera y verano. Canola y chícharo tienen una mayor acumulación de MS que la alfalfa y chicoria durante el ciclo de invierno (Reta et al., 2008). El sistema tradicional avena-maíz-maíz destacó principalmente por el buen rendimiento y contenido energético del maíz; sin embargo, su bajo contenido de proteína se reflejó en un menor rendimiento de forraje y en productividad del agua para la producción de PC (Cuadro 2).

Entre los sistemas de especies alternativos evaluados, sobresalió el que incluyó canola, maíz y soya pues produjo rendimientos de PC similares a los de alfalfa, pero con mayores rendimientos de MS (62 \%) y ENL (77 $\%)$, así como una mayor PA en cuanto a MS (60\%) y ENL (75\%) (Cuadro 2). Esta respuesta se relacionó con el forraje de buena calidad aportado por canola y soya, los cuales se caracterizan por su alto contenido de proteína y energía (Cuadro 1). El sistema chícharo-maízsoya también superó a la alfalfa en el rendimiento y PA de MS y ENL, pero la menor concentración de PC en el chícharo respecto a canola y alfalfa, redujo significativamente su rendimiento de MS y PA en la producción de PC (Cuadro 2).

En conclusión, la incorporación de especies alternativas, como canola, chícharo y soya en los sistemas de producción forrajeros, que incluyan como cultivo principal al maíz, permite disponer de sistemas alternativos a la alfalfa, con valores similares o algo menores de rendimiento y productividad del agua de PC pero con mayores valores en cuanto a rendimientos de MS y ENL.

\section{AGRADECIMIENTOS}

Al INIFAP, Fundación Produce Coahuila, A.C. y PIFSV por el financiamiento de este estudio.

\section{BIBLIOGRAFÍA}

Bremner J M (1996) Nitrogen-total. In: Methods of Soil Analysis: Part 3. D L Sparks (ed). SSSA Book Ser. 5. SSSA, Madison, WI. pp:1085-1121.

Goering H K, P J Van Soest (1970) Forage Fiber Analysis (apparatus, reagents, procedures, and some applications). Handbook 379. USDA-ARS, Washington, DC. 20 p.

Hudson N W (1997) Medición Sobre el Terreno de la Erosión del Suelo y de la Escorrentía. Boletín de Suelos de la FAO-68. Silsoe Associates. Ampthill, Bedford, Reino Unido. 147 p.

NRC, National Research Council (2001) Nutrient Requirements of Dairy Cattle. Natl. Acad. Press, Washington, D.C. 408 p.

Núñez H G, A Peña R, F González C, R Faz C (2006) Características de híbridos de maíz de alto rendimiento y calidad nutricional de forraje. In: Maíz Forrajero de Alto Rendimiento y Calidad Nutricional. J G Martínez R, Y I Chew M, I Reyes J, G Núñez H (eds). Libro Científico Núm. 3. INIFAP-CIRNOCCELALA. pp:45-97.

Putnam D H, P Robinson, E DePeters (2008) Forage quality and testing. In: Irrigated Alfalfa Management in Mediterranean and Desert Zones (Chapter 16). C G Summers, D H Putnam (eds). Oakland, University of California. A-N-R Publ. 8302. pp:2-25.

Quiroga G H M, R Faz C (2008) Incremento de la eficiencia en el uso del agua por la alfalfa mediante la suspensión de riegos en el verano. Terra Latinoam. 26:111-117.

Reta S D G, J S Serrato C, R Figueroa V, J A Cueto W, S Berumen P, J Santamaría C (2008) Cultivos Alternativos con Potencial 
de Uso Forrajero en la Comarca Lagunera. Libro Técnico Núm. 3. INIFAP-CIRNOC-CELALA. $268 \mathrm{p}$.

Russell A E, D A Laird, A P Mallarino (2006) Nitrogen fertilization and cropping system impacts on soil quality in Midwestern Mollisols. Soil Sci. Soc. Am. J. 70:249-255.
SAS Institute (1985) SAS User's Guide. Statistics, version 5.0. $5^{\text {th }}$ Ed. SAS Inst., Cary, NC. 956 p. 